\title{
Guerres des manuels scolaires en Hongrie
}

Querelles sur le pluralisme et enjeux idéologiques

School textbook wars in Hungary. Quarrels on pluralism and ideological stakes Guerras de manuales escolares en Hungría. Contiendas sobre el pluralismo y los retos ideológicos

\section{Iván Bajomi}

\section{CpenEdition}

\section{Journals}

Édition électronique

URL : https://journals.openedition.org/ries/6151

DOI : $10.4000 /$ ries.6151

ISSN : 2261-4265

Éditeur

France Education international

\section{Édition imprimée}

Date de publication : 30 avril 2018

Pagination : 123-133

ISBN : 978-2-85420-618-0

ISSN : $1254-4590$

Référence électronique

Iván Bajomi, "Guerres des manuels scolaires en Hongrie », Revue internationale d'éducation de Sèvres

[En ligne], 77 | avril 2018, mis en ligne le 30 avril 2020, consulté le 25 juin 2021. URL : http://

journals.openedition.org/ries/6151; DOI : https://doi.org/10.4000/ries.6151 


\title{
Guerres des manuels scolaires en Hongrie
}

\section{Querelles sur le pluralisme et enjeux idéologiques}

\author{
Iván Bajomi \\ Université Eötvös Lóránd (ELTE), Hongrie
}

Depuis le début des années 1980, on constate en Hongrie de très forts mouvements de balancier, en matière de régulation des contenus scolaires. Sous le régime finissant de János Kádár, à la suite de l'action réformatrice de chercheurs en éducation et des politiciens de l'aile réformiste du parti unique, la marge de manœuvre des enseignants s'était élargie. Dès 1985, les services d'inspection furent transformés en instituts pédagogiques départementaux censés conseiller et non contrôler le travail des enseignants. Selon le professeur d'histoire Pivárcsi (1998), de nombreux enseignants pouvaient éviter, après 1985, de s'en tenir aux programmes et aux manuels d'histoire sans s'y opposer ouvertement :

La majorité des enseignants s'efforçaient d'enseigner les évènements les plus discutés en se basant uniquement sur les faits et en évitant les approches idéologiques, voire en omettant tout simplement d'enseigner l'histoire hongroise postérieure à 1945.

Un proviseur d'un lycée de Budapest a évoqué de la façon suivante les pratiques enseignantes au cours de cette période :

Ils ont préféré raconter par leurs propres mots la révolution de 1956 ou recourir à des articles de presse [contemporains] dans la mesure où ce qui figurait dans les manuels n'était pas conforme à la réalité. ${ }^{1}$

\section{QUERELLE AUTOUR DES MANUELS DE LITTÉRATURE ANCIENS ET MODERNES}

Durant les premières décennies du régime communiste, les enseignants devaient utiliser des manuels uniques. Une étude de Gyürei (1981) montre toutefois qu'une partie des enseignants, probablement ceux qui étaient les mieux dotés en capital culturel, pouvaient se permettre, dès le milieu des années 1970, de ne pas utiliser les manuels officiels et obligatoires :

Certaines expériences montrent qu'en enseignant les professeurs ne s'appuient pas sur les manuels. Je pense que cela n'est vrai que partiellement, puisqu'il serait erroné d'avoir uniquement en vue une seule étroite couche. Parmi les

1. « Legfeljebb nem tankönyvből tanítunk » [Dans le pire des cas, nous n'utiliserons pas de manuels pour enseigner]. hvg.hu, 12 décembre 2013 [https://goo.gl/RZpFKQ] 
professeurs très nombreux sont qui préparent leurs cours en se basant en premier lieu sur le manuel, et les élèves apprennent aussi de celui-ci puisqu'il est plus facile et aussi plus sûr de s'approprier ainsi ce qui doit être appris. » (Gyürei, 1981, p. 115)

L'étude de Gyürei illustre aussi parfaitement à quel point les manuels de certaines disciplines étaient imbibés de contenus idéologiques sous le communisme. En analysant les représentations relatives à la vie et l'œuvre de l'écrivain Attila József (1905-1937), l'auteure a démontré que, pendant plusieurs décennies, les manuels scolaires ont présenté ce poète comme un fervent communiste, alors que, au cours de ses dernières années, il avait adopté un point de vue très critique à l'égard du mouvement communiste.

Si l'utilisation de manuels uniques avait prévalu au début du régime communiste, au cours de ses dernières années, le choix entre plusieurs manuels devint possible pour certaines disciplines jugées moins importantes du point de vue idéologique, comme c'était le cas de l'apprentissage de la lecture. Ajoutons à cela, pour la petite histoire et pour illustrer les forts liens de dépendance entre les concepteurs de la politique éducative que la Maison d'édition des manuels, une entreprise d'État jouissant du monopole des manuels scolaires, était logée au cinquième étage d'un bâtiment... dont les quatre autres niveaux étaient occupés par le ministère de l'éducation.

En ce qui concerne l'enseignement de la littérature, un important changement se produisit à la fin des années 1970, lorsque des chercheurs de l'Institut de littérature de l'Académie des sciences entreprirent de rédiger une série de manuels de littérature. Tandis que, durant les premières décennies du communisme, les auteurs des manuels étaient désignés par la Maison d'édition des manuels, à partir de 1976, des appels d'offre furent lancés. Ces chercheurs avaient été choisis pour rédiger les manuels des quatre classes du lycée. Les trois premiers volumes parus étaient novateurs à maints égards. Des œuvres du canon littéraire communiste (par exemple, un roman de Gorki) furent mis de côté, tandis que les manuels mentionnaient des auteurs comme Joyce et Beckett, autrefois décriés par les gardiens de l'orthodoxie marxiste en tant que représentants de la littérature bourgeoise ou décadente. Les auteurs des manuels tentèrent également de faire connaître de nouvelles approches et de nouvelles recherches. C'est pourquoi l'utilisation de ces ouvrages exigeait un travail considérable de la part d'enseignants qui n’étaient pas informés des résultats des recherches menées en Occident, entres autres sous l'égide du structuralisme. András Veres, l'un des auteurs de ces manuels, déclara ainsi à la radio au sujet de la pratique des enseignants :

L'opinion publique des professeurs est loin d'être unanime [...]. Nombreux sont ceux qui sont contents de l'ouverture dont fait preuve le manuel, satisfaits de jouir d'une certaine liberté, de pouvoir faire des choix [...]. Mais d'autres ne sont pas contents de cela, ils ne veulent pas bénéficier de telles possibilités (...). J'ai aussi entendu parler d'un collègue qui, au vu du résultat final, a pu vaincre 
son hostilité et ses appréhensions initiales. [...] Certaines personnes ne se servent pas du tout du nouveau manuel, voire interdisent carrément son utilisation. [...] On a même parlé de haute trahison au sujet du manuel (...) en prétendant que la cause de la littérature hongroise avait été trahie. » (Pála, 1991)

À la suite de la publication des trois premiers volumes, des critiques violentes furent émises, notamment au sein de l'organe central du Parti communiste. Leurs auteurs prétendaient que ces livres véhiculaient une vision du monde pessimiste et trop individualiste, et protestaient également contre le fait que la littérature hongroise y occupait moins de place que dans les manuels précédents. Après la parution de nombreuses critiques similaires, les auteurs ne purent mener à bien leur tâche et finalement les lycéens de terminale durent acheter un manuel moins novateur, qu'un professeur de lycée avait initialement rédigé pour les lycées professionnels.

Sans pouvoir présenter tous les méandres des débats provoqués par les volumes successifs des manuels de littérature, signalons qu'en 1982, c'est-à-dire presque une décennie avant l'effondrement du régime communiste, l'écrivain István Csurka avait ouvertement évoqué dans un quotidien la nécessité d'autoriser la pluralité des ouvrages scolaires :

Il est impossible de bien concevoir le seul et unique manuel salvateur. [...] Là aussi nous avons affaire à un ancien et mauvais mode de régulation poussé à l'extrême que l'on devrait rapidement dépasser. Il faudrait que plusieurs ouvrages scolaires puissent être rédigés parallèlement afin que les écoles, voire les enseignants puissent choisir parmi eux [...]. (Pála, 1991)

\section{LE DifFICILE AVÈnEMENT DE LA PLURALITÉ DES MANUELS SCOLAIRES}

Le processus de libéralisation entamé en Hongrie dans le domaine de l'éducation bien avant la chute du mur de Berlin s'amplifia après la mise en place d'un régime politique multipartite entre 1989 et 1990.

D’une part, la régulation des contenus avait été fortement modifiée, avec le passage d'un curriculum scolaire unifié à une régulation basée sur un curriculum national de base qui laissait une autonomie considérable aux écoles pour élaborer leur propre programme. Dès les premiers débats concernant le degré d'autonomie à laisser aux établissements scolaires, on constata une forte opposition entre les acteurs (Bajomi, 2003). Ceux qui se situaient à la gauche de l'éventail politique étaient enclins à accorder une large marge de manœuvre aux établissements, qui aurait permis aux enseignants de regrouper - s'ils le souhaitaient - les matières scolaires traditionnelles en blocs de connaissances rassemblant plusieurs disciplines. Par exemple, au lieu d'enseigner séparément la physique, la chimie et la biologie, etc., les équipes enseignantes pouvaient choisir de remplacer ces disciplines par une nouvelle matière intitulée 
« connaissance de la nature », dont les objectifs pouvaient varier d'un établissement à l'autre. ${ }^{2}$ En revanche, les gouvernements de droite se montraient favorables à une régulation plus stricte, symbolisée notamment par l'introduction de programmes cadres définissant clairement les matières scolaires. Cela fut le cas durant la première coalition de droite au pouvoir, entre 1990 et 1994, puis entre 1998 et 2002, lorsque V. Orbán dirigeait une coalition de droite. ${ }^{3}$

La liberté des enseignants et des équipes pédagogiques se trouva également considérablement élargie dans le domaine des manuels. Toutefois après les premières élections libres de 1990, on vit resurgir chez les nouveaux dirigeants du pays des velléités d'exercer une influence idéologique, par le biais des manuels scolaires de trois disciplines (histoire, géographie et littérature), considérées traditionnellement par les élites politiques comme particulièrement importantes du point de vue de la création d'une identité nationale.

Au début des années 1990, le ministère de l'éducation et de la culture du nouveau gouvernement de droite lança un appel d'offre pour un nouveau manuel d'histoire. Comme personne n'y répondait, une association demanda à des historiens d'obédience socialiste ou libérale de rédiger un manuel d'histoire destiné aux terminales de lycée. Les responsables ministériels homologuèrent celui-ci, puis - selon la rumeur, sous l'influence du principal parti gouvernemental - firent savoir à plusieurs reprises que ce manuel "était inacceptable». Cela entraîna un scandale dans la presse. Il faut noter qu'au même moment, la toute jeune Association des professeurs d'histoire s'était prononcée en faveur de la pluralité des manuels dans les termes suivants :

Tous les manuscrits que la profession considère comme bons et aptes à être utilisés en classe doivent pouvoir être inscrits sur la liste des manuels, tandis que des points de vue extraprofessionnels ne doivent en aucune façon influencer les décisions. ${ }^{4}$

Le contenu de cette citation est en rupture avec l'idée traditionnelle selon laquelle il n'existerait qu'une seule histoire, voire un seul grand récit historique que l'on devrait tenir pour vrai. Nagy (1994) a analysé de la façon suivante les leçons de cette affaire :

\footnotetext{
2. Les expérimentations locales, peu nombreuses et fort diverses, avaient en commun une volonté des enseignants de développer la curiosité des enfants à l'égard des phénomènes de la nature, notamment par le biais d'expériences. Les professeurs des diverses matières qui se regroupaient afin d'assurer un enseignement interdisciplinaire s'efforçaient de dégager avec les élèves les principales caractéristiques communes des différentes démarches scientifiques visant à modéliser les phénomènes naturels. Il serait toutefois exagéré de prétendre que les enseignants accordaient ainsi une importance particulière aux préoccupations épistémologiques, ne serait-ce que parce que dans certains cas, ces cours s'adressaient à des élèves âgés de 12 ans seulement.

3. Au moment du changement de régime, V. Orbán a dirigé un parti libéral. Après quelques années, sa formation politique est devenue, sous sa direction, le principal parti de la droite hongroise et a quitté l'Internationale libérale pour adhérer en 2000 au Parti populaire européen (PPE), qui rassemble les partis conservateurs du continent. Récemment, plusieurs dirigeants du PPE ont suggéré d'exclure le Fidesz, le parti de M. Orbán, en raison de ses prises de positions jugées extrémistes.

4. "A történelemtanárok 2. országos konferenciájnak ajánlásai » (Recommandations de la seconde conférence nationale des enseignants d'histoire ), 1992 [https://goo.gl/PNHjVy].
} 
Ce scandale est important du point de vue de la politique des manuels scolaires. En effet, la conception de ce manuel était conforme à tous les critères que l'on peut imaginer dans le cas d'un tel processus. L’ouvrage a été rédigé par des historiens qui étaient des spécialistes de la période et en tant que candidats ou docteurs ès sciences réputés détenaient les titres scientifiques formellement requis, tout en jouissant d'une légitimité informelle. Par ailleurs, ce ne sont pas des forums d'historiens qui ont remis en question leurs affirmations scientifiques, mais l'appareil d'État ou la politique. Contrairement à tous les manuels précédents, la transformation du texte en manuel scolaire s'est faite d'une façon exemplaire: des dizaines d'élèves ainsi que des enseignants en poste et des spécialistes en méthodologie ont débattu à plusieurs reprises du manuel (...). Ils ont modélisé en quelque sorte préalablement les éventuels conflits professionnels et par ce biais les ont éliminés à l'avance. Les lecteurs de la Maison d'édition des manuels ont accepté l'ouvrage, que le ministère a homologué. Cela peut être considéré comme une préfiguration du processus d'homologation qui apparaîtra ultérieurement dans la loi comme une activité placée sous l'égide du Conseil national de l'éducation publique. (Nagy, 1994)

À la lumière d'une étude récente (Knausz, 2015) affirmant que la plupart des anciens manuels d'histoire constituent des documents véhiculant un grand récit historique, on serait tenté de formuler l'hypothèse que le manuel en question était aussi un document de cet ordre. Autrement dit, il véhiculait, sous la forme d'un grand récit, une vision relativement cohérente des conceptions historiques partagées par les élites de gauche de la Hongrie contemporaine, tandis que les représentants du ministère auraient souhaité que l'on utilise en terminale un livre d'histoire essentiellement inspiré des représentations qu'avaient du passé de la Hongrie les élites de droite. Cette hypothèse semble être confirmée par Nagy (1994) :

Selon les souvenirs concomitants de plusieurs témoins, le porte-parole du ministère de l'éducation et de la culture aurait dit que "le manuel élaboré avec le soutien du ministère doit être conforme à certaines attentes idéologiques ».

Comme, à l'époque, aucun manuel scolaire disponible ne correspondait aux attentes du ministère, celui-ci finança en 1992 la distribution gratuite dans les écoles d'une brochure rédigée par un professeur d'histoire en poste dans un lycée catholique. Selon Nagy, plusieurs passages de ce texte pouvaient être interprétés comme une approbation sans réserve de la politique hongroise officielle de l'entre-deux-guerres, qui avait été menée sous le régime semi-autoritaire de l'amiral Horthy. ${ }^{5}$

Si, au début des années 1990, les décideurs politiques d'obédience conservatrice ne voyaient pas d'un bon oil que les lycéens apprennent l'histoire du $\mathrm{XX}^{\mathrm{e}}$ siècle dans un manuel rédigé par des auteurs proche de l'opposition, les

5. Bornons-nous à signaler que c'est au début de la période pendant laquelle il était gouverneur que fut adoptée en 1921 la première loi anti-juive d’Europe, qui était censée limiter le nombre d'étudiants juifs dans les universités, tandis qu'à partir de la fin des années 1930, toujours sous son mandat, plusieurs autres lois limitant les droits des Juifs furent votées. 
luttes relatives aux manuels d'histoire se sont apaisées dès que plusieurs livres scolaires sont devenus accessibles. Le marché des manuels a pu se développer après l'abolition du monopole de la Maison d'édition des manuels survenue en 1991. Selon les résultats d'une enquête menée auprès des enseignants de la section supérieure de l'école fondamentale, au début des années 2000, pour chaque promotion, huit ou neuf manuels différents étaient utilisés, et trois d'entre eux étaient plébiscités par environ $90 \%$ des professeurs d'histoire (Csabai, 2002). Si l'ouvrage qui rassemble les documents relatifs aux conflits suscités par les nouveaux manuels de littérature a été intitulé à bon droit Guerre des manuels (Pála, 1991), à propos des manuels d'histoire utilisés dix ou douze ans après le changement de régime, il est tout à fait légitime de parler d'une coexistence relativement pacifique. En 2010, quelques semaines avant l'arrivée au pouvoir du second gouvernement Orbán a eu lieu une réunion consacrée aux manuels d'histoire, dont Katona (2010) a parlé en ces termes :

Elle s'est déroulée dans une ambiance constructive, voire amicale et [...] malgré la présence des auteurs, les débats ont été exempts de toute querelle personnelle.

Dans ce compte rendu, on apprend aussi qu'à l'exception d'un manuel déjà utilisé au début des années 1990, les livres d'histoire plus récents contenaient non seulement des fragments provenant de sources historiques mais aussi un nombre important d'illustrations (photos, caricatures, données quantifiées et cartes), et plusieurs auteurs avaient également formulé des questions visant à développer une réflexion autonome chez les élèves. Certains manuels invitaient même les élèves à procéder à des recherches au sujet de faits historiques controversés. À cette fin on mettait à leur disposition non seulement des sources historiques mais aussi des fragments tirés d'études ou de livres ayant fait l'objet de débats. L'historien hongrois Ottó Szabolcs, "parle à ce sujet de "pluralisme provoqué", qui permet de confronter des points de vue relatifs à telle ou telle question d'histoire et donne ainsi la possibilité aux élèves de procéder à des choix intellectuels, voire de réviser leur point de vue initial. »(Katona, 2010).

Par ailleurs, tous les manuels d'histoire en vente vers la fin des années 2000 ne véhiculaient pas des visions identiques. Ainsi, une étude de László Miklósi ${ }^{6}$, qui compare les représentations des Juifs dans divers manuels d'histoire portant sur l'entre-deux guerres, met en évidence des différences de taille. Un auteur aurait utilisé des termes équivoques pour évoquer le retour de certains territoires perdus par la Hongrie à la suite des accords de Paris, au lendemain de la Première Guerre mondiale, et qui ont pu être à nouveau rattachés au pays grâce aux liens amicaux noués, durant les années 1930, par l'amiral Horthy avec l'Allemagne hitlérienne, tandis que les auteurs d'autres manuels traitaient de façon plus nuancée cette question. 


\section{LA SUPPRESSION RÉCENTE DU MARCHÉ DES OUVRAGES SCOLAIRES}

Contrairement au paysage relativement paisible que nous venons de décrire, on assiste, depuis l'arrivée au pouvoir du second gouvernement de M. Orbán, à une exacerbation des tensions provoquées par la suppression progressive du marché des livres scolaires et l'introduction rapide de nouveaux manuels faisant l'objet de vives critiques.

Depuis 2010, bénéficiant d'une forte majorité parlementaire, les deux gouvernements successifs de M. Orbán ont procédé à de très vastes mesures d'étatisation et de centralisation dans le domaine de l'éducation ${ }^{7}$, et ceci sans concertation avec les autres partis ni avec les groupes d'intérêt concernés.

L'édition des manuels scolaires n'a pas échappé à ce type de mesures. Dans un premier temps, leur diffusion a été placée sous l'égide d'une entreprise d'État, puis on a procédé à la ré-étatisation de la Maison d'édition des manuels précédemment privatisée ; dans le même temps, l'État a acheté la seconde maison d'édition de manuels du pays, acquérant ainsi les droits d'auteurs dont disposait cette entreprise. Puis, en utilisant d'importantes fonds européens, le secrétariat d'État à l'éducation a fait rédiger des livres scolaires expérimentaux sous l'égide de son institut de développement, dans des délais très courts. En parallèle, le marché privé de l'édition scolaire s'est réduit comme peau de chagrin. Par le biais d'une liste des manuels homologués par le ministère et d'une autre liste, plus réduite et totalement illégale, émanant des instances académiques, on a interdit l'achat de manuels « hors liste " aux écoles qui relevaient depuis 1990 des collectivités locales et qui avaient été récemment ré-étatisées. En peu de temps, plusieurs éditeurs ont disparu du paysage scolaire et l'avenir de ceux qui s'y maintiennent est incertain.

Les autorités avancent des arguments variés pour justifier le fait que les enseignants ne pourront choisir qu'entre deux manuels ou n'auront pas le droit de choisir. D'une part, elles insistent sur le fait que parallèlement au passage à ce «nouveau » système, de plus en plus d'enfants reçoivent gratuitement leurs manuels. Il est évident que de ce point de vue, l'État a tout intérêt à limiter ses dépenses dues à la gratuité des manuels scolaires. On invoque aussi la nécessité de créer des manuels favorisant l'utilisation des nouvelles technologies. La suppression du marché de l'édition scolaire est aussi justifiée par des arguments égalitaristes, selon lesquels tous les enfants, même les plus déshérités, ont le droit d'apprendre dans les mêmes manuels que les enfants issus de familles plus riches. À ce sujet, László Arató, président de l'Association des professeurs de hongrois, a déclaré dans un entretien :

7. Pour une description plus détaillée des répercussions éducatives du tournant autoritaire survenu en Hongrie depuis 2010, voir Bajomi, 2017. 
Prétendre que des manuels uniques favorisent l'égalité des chances témoigne d'une ignorance inouïe. Un manuel peut convenir à un enfant et peut être mauvais dans le cas d'un autre. (...) Ce sont les supports conçus en fonction des caractéristiques des différents groupes et ou individus qui favorisent l'égalité des chances. ${ }^{8}$

Dans un premier temps, seules les organisations professionnelles et les syndicats d'enseignants, ainsi que les partis d'opposition, ont critiqué les mesures allant dans le sens du rétrécissement et éventuellement de la suppression totale du marché des manuels scolaires. Notons aussi que certains enseignants, craignant les éventuelles mesures répressives des nouvelles autorités académiques dépendant du Centre Klebelsberg, ont réagi à la situation en utilisant une double stratégie. Ainsi, en 2014, Index, l'un des sites Internet les plus lus du pays, a écrit au sujet des nouveaux manuels :

Bien que dans les écoles d'État, seuls les manuels expérimentaux et autres manuels recommandés puissent être utilisés, en réalité, dans beaucoup d'endroits, les enseignants récalcitrants (ou plutôt autonomes) ne les utilisent pas pour enseigner. Ils ont fait acheter aux parents les anciens manuels bien rodés et non conseillés par le Centre Klebelsberg ${ }^{9}$.

Le même article précise ceci, au sujet d'une école de province :

Les parents collectionnent dans un entrepôt secret les manuels de la maison d'édition Mozaik, qui ont été ôtés de la liste des manuels, et si l'année prochaine il $\mathrm{y}$ a de la place dans la bibliothèque de l'école, on les y déposera. ${ }^{10}$

Sur un forum de parents, on peut lire cette plainte plus récente :

Les enfants doivent faire acheter [par leurs parents] beaucoup de matériaux supplémentaires ou faire faire beaucoup de photocopies car le contenu des manuels ne convient pas. Dans certaines classes, les enfants ne s'appuient pour apprendre que sur ce que le professeur leur a dicté. ${ }^{11}$

Les nouveaux manuels, très rapidement conçus, furent régulièrement et fortement critiqués, tant à cause d'erreurs factuelles qu'en raison de contenus erronés, reflétant même parfois des modes de pensée d'une autre époque. (Faute de place, nous nous contenterons de mentionner le cas d'un manuel de sciences naturelles destiné à des enfants de 11 ans, selon lequel les garçons seraient plus doués que les filles pour les mathématiques.)

8. Teczár Sz. (2014) : «Az ember államosiítása - Arató László a tankönyvbotrányról » [L'étatisation de l'homme - entretien avec László Arató au sujet du scandale des manuels scolaires], Magyar Narancs, 17 avril, [https://goo.gl/Bgfy88] 9. Ce centre a été créé à la suite de la ré-étatisation des écoles. Portant le nom d'un ministre de l'éducation du régime semi-autoritaire de l'entre-deux guerres, il est chargé de gérer les aspects pédagogiques du fonctionnement des écoles d'État. Cette administration fait l'objet de critiques récurrentes depuis sa création, notamment en raison de son mode de fonctionnement lent et opaque, ainsi que de l'insuffisance chronique des ressources qu'elle alloue aux écoles.

10. Joób S., Halmos M., Kolozsi Á. (2014) : « Sutyiban más tankönyvből tanítanak a tanárok » (Les enseignants utilisent en catimini d'autres manuels), Index, 27 novembre [https://goo.gl/q5V29y].

11. " "Tehetetlen dühömben legszívesebben sikítanék” - szülők a tankönyvekről », [" “’’aimerais hurler pour faire sortir de moi cette colère impuissante" - des parents au sujet des manuels »], Szülöi hang, 26 avril 2017, [https:// goo.gl/zkzKZt]. 
En dehors des formes de contestation mentionnées et qui restent finalement peu visibles, les velléités gouvernementales visant à supprimer le marché des manuels ont été critiquées dans une lettre de doléances adressée par les enseignants du lycée Ottó Herman de la ville de Miskolc, en novembre 2016, au nouveau centre académique Klebelsberg. Parmi les critiques sévères formulées au sujet du mode de gouvernance mis en œuvre dans le domaine de l'éducation, la question des manuels a été évoquée :

La nouvelle génération des manuels est désignée par le terme "manuels expérimentaux », c'est à dire faisant l'objet d'une expérimentation $[\ldots]$, tandis que des livres scolaires de très haut niveau ont été abandonnés. (...) Lorsque le système scolaire procède à des expérimentations, on fait des expériences sur les enfants. ${ }^{12}$

Ces doléances étant restées sans réponse, deux mois plus tard, le 6 janvier 2017, la lettre a été publiée sur un site local, ce qui a déclenché un vaste mouvement national des enseignants, qui s'est propagé en Hongrie à toute vitesse. Si la vague de protestation a incité les autorités à désamorcer ce mouvement par la création d'une nouvelle instance consultative, les diverses organisations et associations qui ont soutenu le mouvement des enseignants n'ont pas rejoint cette instance, car elles n'approuvaient ni sa composition ni son mode de fonctionnement unilatéral. Elles ont préféré créer une nouvelle organisation de coordination regroupant une soixantaine d'organisations, appelée Plateforme civile de l'éducation publique. Quelques semaines plus tard, parmi les revendications immédiates de la plateforme, on pouvait lire dans le point 6 :

Nous exigeons de pouvoir réellement bénéficier du libre choix des manuels ! Il faut lever toutes les entraves limitant l'utilisation des manuels scolaires et des "kits pédagogiques » qui sont basés sur les compétences ${ }^{13}$ et qui ont été développés durant de longues années. Il faut que le gouvernement et le Centre Klebelsberg cessent de se référer à la liste des manuels obligatoires et de recourir à tout autre mode illégal d'intervention.

Notons que, parallèlement aux mobilisations des enseignants, des mouvements d'élèves sont également nés. Lors d'un forum, un jeune a cité comme l'une des principales lacunes des enseignements assurés dans les écoles le fait que l'on n'y développe pas le sens critique ni la créativité des élèves. ${ }^{14}$

12. Nyílt levél a Herman Ottó Gimnáziumból [Lettre ouverte du Lycée Ottó Herman], 2016 [https://goo.gl/JZBh2D]. 13. "Megjelent a tiltakozó tanárok 12 pontja » [Les 12 points des enseignants contestataires ont été rendus publics], $1^{\mathrm{er}}$ mars 2016 [https://goo.gl/RKirhS]. Les outils pédagogiques ont été conçus entre 2002 et l'arrivée au pouvoir en 2010 du second gouvernement Orbán, entre autres sur la base des recommandations concernant les compétences clés définies par un groupe de travail de la Commission européenne. Il s'agissait de développer les facultés des enfants dans huit domaines : éducation préscolaire, faculté d'interpréter des textes et compétences rédactionnelles, compétences en mathématiques et en logique, communication en langues étrangères, compétences sociales et environnementales, aptitude à gérer sa carrière personnelle et compétences numériques.

14. Bécsi O. (2016) : Tapsvihar közepette csatlakoztak a diákok a tanártüntetéshez [Les lycéens ont rejoint le mouvement des enseignants sous de vives acclamations] mno.hu, 14 mai [https://goo.gl/PUupkL] 
Le mouvement déclenché en janvier 2016 a notamment eu pour résultat une grève importante des enseignants hongrois, soutenue par les syndicats de l'éducation, ainsi qu'un mouvement enseignant autonome appelé "J'aimerais pouvoir enseigner ", dont le nom et les diverses prises de position orales et écrites témoignent d'une volonté de pouvoir enseigner librement, sans entraves. La grève a eu lieu le 20 avril, tandis que la Plateforme civile de l'éducation publique a présenté en décembre 2017 un programme complet de politique éducative, qui comporte la phrase suivante: "Il faut recréer un marché des manuels et des autres supports pédagogiques. » ${ }^{15}$

Les auteurs de ce programme auraient aimé pouvoir discuter avec les représentants du gouvernement de leurs propositions, mais ces derniers ont déclaré que seule l'instance consultative créée sous l'égide du secrétariat d'État, en février 2016, pouvait servir de cadre aux négociations. Puis Szilárd Németh, le vice-président du parti Fidesz, que dirige M. Orbán, est allé plus loin en déclarant, début 2017, qu'il faudrait nettoyer la Hongrie du mouvement « J'aimerais pouvoir enseigner» et de l'Association des professeurs d'histoire, puisque ces organisations poursuivent des objectifs politiques en critiquant régulièrement la politique éducative du gouvernement. Ce qui veut dire que seul le gouvernement a le droit de s'exprimer au sujet de la politique éducative.

La radicalisation du milieu enseignant hongrois contemporain semble confirmer la thèse d'un politologue américain (Gurr, 1970) selon lequel ce ne sont pas nécessairement les plus démunis qui se révoltent les premiers, mais souvent ceux dont certaines aspirations (par exemple la possibilité de choisir parmi plusieurs manuels) ont pu être satisfaites à un moment et qui constatent ultérieurement une dégradation de leur situation ou des conditions dans lesquelles ils travaillent.

\section{BibLIOgRAPHIE}

BAJOMI I. (2003) : "Un jeu de balancier. La définition réglementaire des contenus d'enseignement dans la Hongrie post-communiste ", Revue Internationale d'éducation de Sèvres, $\mathrm{n}^{\circ}$ 32, p. 42-50. [http://journals.openedition.org/ries/1818 ; DOI : 10.4000/ ries.1818]

BAJOMI I. (2017) : Réactions suscitées par les mesures éducatives d'un régime de plus en plus autoritaire. Le cas de la Hongrie, Éducation et sociétés, n 39, p. 35-51.

CSALA I. (2002) : [La situation en 2002 de la discipline d'histoire selon les résultats d'une enquête] : [https://goo.gl/xcxSDc]

GURR T. (1970) : Why Men Rebel?, Princeton, Princeton University Press, 1970.

15. « Kockás könyv - kiút az oktatási katasztrófából » [Livre à carreaux - Comment mettre fin à la situation catastrophique de l'enseignement], Civil Közoktatási Platform, 2016 [https://goo.gl/edchNb] 
GYÜREI V. (1981) : A fanatikus optimista - József Attila a tankönyvek tükrében [Un optimiste fanatique - Attila József à la lumière des manuels], Medvetánc, $\mathrm{n}^{\circ}$ 2-3, p. 101-116.

KATONA A. (2010) : Tankönyvvita a 12. osztályos történelemkönyvekról [Débat sur les manuels scolaires de l'année terminale du lycée], Elektronikus Könyv és Nevelés, vol. 12, n 3 [http://www.matarka.hu/cikk_list.php?fusz=99842]

KNAUSZ I. (2015) : A múlt kútjának tükre A történelemtanitás céljairól [Le miroir du puits du passé - réflexions sur les objectifs de l'enseignement de l'histoire], Miskolc, Miskolci Egyetemi Kiadó.

NAGY P. T. (1994): Tankönyvpolitika a kilencvenes években (Politique des livres scolaires des années quatre-vingt-dix), Education, vol. 3, n 4, p. 519-534.

PIVÁRCSI I. (1998) : A történelemtanítás aktuális problémai [Problèmes actuels de l'enseignement de l'histoire], Iskolakultúra, $\mathrm{n}^{\circ} 11$, p. 47-59.

PÁLA K. (coord.), (1991): Tankönyvháború : viták a gimnáziumi irodalomoktatás reformjáról a hetvenes-nyolcvanas években [Guerre des manuels : Débats relatifs à la réforme de l'enseignement de la littérature au lycée], Budapest, MTA Irodalomtudományi Intézete - Argumentum. 
